\title{
Sources d'influence de l'engagement des étudiants dans un dispositif de classe inversée à l'université : le cas de PedagInnov
}

Laetitia Gerard and Ariadna Ayala Rubio

\section{(2) OpenEdition Journals}

Electronic version

URL: http://journals.openedition.org/ripes/2212

DOI: $10.4000 /$ ripes.2212

ISSN: 2076-8427

Publisher

Association internationale de pédagogie universitaire

\section{Electronic reference}

Laetitia Gerard and Ariadna Ayala Rubio, «Sources d'influence de l'engagement des étudiants dans un dispositif de classe inversée à l'université : le cas de PedagInnov », Revue internationale de pédagogie de l'enseignement supérieur [Online], 36(1) | 2020, Online since 12 April 2020, connection on 08 September 2020. URL : http://journals.openedition.org/ripes/2212 ; DOI : https://doi.org/10.4000/ripes.2212

This text was automatically generated on 8 September 2020

Article L.111-1 du Code de la propriété intellectuelle. 


\title{
Sources d'influence de l'engagement des étudiants dans un dispositif de classe inversée à l'université : le cas de PedagInnov
}

\author{
Laetitia Gerard and Ariadna Ayala Rubio
}

\section{Introduction et contexte}

1 Le Projet PedagInnov est une expérimentation de classe inversée à l'université. Il s'inscrit dans le cadre de l'Initiative d'Excellence en Formations Innovantes (IDEFI) Individualisation, Diversification, Évaluation et Accompagnement (IDEA) porté par la Communauté d'universités et établissements (COMUE) de l'Université Paris-Est. Dix enseignants universitaires volontaires ont accepté de participer à ce projet en réorganisant leurs cours selon le principe de la classe inversée ${ }^{1}$. À l'issue de l'expérimentation, PedagInnov a fait l'objet d'une étude-évaluation par la première auteure de cet article, qui a abouti à la rédaction d'un rapport public, consultable en ligne (Gerard, 2018). Le présent article est issu des résultats de cette étude.

2 PedagInnov est né de cinq constats de la part des dix enseignants expérimentateurs: 1) les étudiants sont plutôt passifs en cours; 2) les étudiants participent peu ou pas; 3) les étudiants fournissent peu de travail personnel et se contentent bien souvent de la mémorisation de leurs notes de cours; 4) la qualité de l'apprentissage tend à rester en surface, ce qui ne favorise pas le développement de connaissances et de compétences transférables dans le milieu professionnel; 5) il y a une faible rentabilité des séances en présentiel, qui sont plutôt utilisées à des fins de transmission des connaissances.

À partir de ces constats, les enseignants volontaires ont choisi d'expérimenter la classe inversée parce qu'elle constitue un moyen d'impliquer davantage les étudiants en cours. En effet, dans une classe inversée le temps de cours en présentiel est optimisé grâce à des activités engageant les étudiants dans une expérience d'apprentissage en 
profondeur (Dumont et Berthiaume, 2016). Bien que différentes formes de classes inversées existent, leur schéma général est similaire. Dans une classe inversée, la transmission des connaissances qui se déroulait généralement en classe se fait désormais chez soi, et le temps d'apprentissage qui avait lieu chez soi est organisé en classe (Dumont et Berthiaume, 2016; Lebrun, 2016; Nizet, Galiano et Meyer, 2016). Ce même schéma a été adopté par les dix enseignants impliqués dans le Projet PedagInnov. En amont du cours, un travail préliminaire était demandé aux étudiants. Dans un premier temps, les étudiants devaient prendre connaissance du cours à l'aide des lectures, du visionnage de diapositives commentées, de MOOC ${ }^{2}$ existants ou de vidéos. Dans un deuxième temps, les étudiants étaient amenés à produire un travail, comme répondre à un questionnaire à choix multiples $(\mathrm{QCM})$ ou réaliser un exercice. En classe, l'enseignant organisait des activités permettant la mise en application des connaissances, prévoyait des exercices, organisait des questions/débats, des projets, des exposés ou du travail d'approfondissement en groupe (Chevalier et Adjedj, 2014).

La classe inversée mise en place dans le cadre du projet PedagInnov constitue une innovation pédagogique telle que définie par Béchard et Pelletier (2001): «l'innovation est une activité délibérée qui tend à introduire de la nouveauté dans un contexte donné et elle est pédagogique parce qu'elle cherche à améliorer substantiellement les apprentissages des étudiants en situation d'interaction et d'interactivité » (Béchard et Pelletier, 2001, p. 133). À partir de cette définition, nous retenons trois termes clés qui définissent une innovation pédagogique: "nouveauté ", "amélioration ", «interaction/interactivité ». Premièrement, l'introduction de la classe inversée à l'université Paris-Est constitue une "nouveauté ", à la fois pour les étudiants et pour les enseignants. La classe inversée représente en effet un " éloignement de la norme » (Berthiaume, 2011), c'est-à-dire un éloignement des pratiques pédagogiques traditionnelles des enseignants de cet établissement. Deuxièmement, PedagInnov vise une "amélioration» des apprentissages puisque le Projet a été initié sur la base des cinq constats évoqués précédemment. Troisièmement, dans la classe inversée telle qu'elle est mise en place par les enseignants de Paris-Est, les étudiants sont mis en situation d'« interaction » et d' « interactivité » lors des séances de travail en classe.

5 Comme pour toutes les innovations pédagogiques, la classe inversée engendre un changement de paradigme pédagogique qui peut être déstabilisant pour l'étudiant. On passe du "paradigme de l'enseignement» dans lequel l'enseignant transmet des connaissances, " au paradigme de l'apprentissage », dans lequel l'étudiant acquiert des compétences, des connaissances de haut niveau, avec une grande part d'autonomie dans la formation. Il y a donc une évolution dans son rapport au savoir et à l'enseignant (Bédard et Béchard, 2009). Dans la classe inversée, la relation pédagogique enseignantenseigné et le rôle de chacun évolue. On attend désormais de l'étudiant qu'il adopte une posture plus active et proactive dans son apprentissage. L'enseignant, quant à lui, se positionne davantage comme un accompagnateur ou un facilitateur. Ainsi, pour réussir, l'étudiant doit faire évoluer son « métier d'étudiant » (Coulon, 1997), ce qui implique de percevoir qu'il y a une évolution attendue dans son métier d'étudiant, vouloir le faire évoluer, savoir comment le faire évoluer et disposer des outils et ressources internes et externes nécessaires pour le faire évoluer. Face à ce changement de paradigme pédagogique, trois profils d'étudiants peuvent émerger (Bédard et Béchard, 2009). Certains étudiants vont s'engager dans l'innovation, en parvenant à faire évoluer leurs stratégies d'apprentissage et en s'adaptant aux nouvelles attentes de l'enseignant. D'autres adopteront une attitude plus attentiste, soit parce qu'ils seront trop 
déstabilisés et ne parviendront pas à faire évoluer leur métier d'étudiant, soit parce qu'ils ne souhaiteront pas s'engager dans l'innovation. Enfin, d'autres adopteront une attitude de rejet par peur de l'inconnu ou par crainte que l'innovation nuise à leurs apprentissages (Bédard, 2006; Bédard et Béchard, 2009; Dalle et Lachiver, 2003).

Or, l'une des conditions essentielles de la réussite de la mise en œuvre d'une innovation pédagogique telle que la classe inversée est précisément l'engagement des étudiants. Une attitude attentiste ou de rejet de la part des étudiants mettrait à mal l'ensemble du dispositif. Par exemple, si les étudiants ne prennent pas connaissance du cours en amont, il leur sera difficile d'appliquer ces connaissances via les activités proposées en classe. L'enseignant serait alors contraint de reprendre l'intégralité du cours en classe, déconstruisant de fait le principe même du dispositif. Cette problématique a été soulevée par Nizet et al. (2016): «l'engagement des étudiants en amont du cours, en autoapprentissage, est parfois très faible et ce manque d'engagement n'est pas encore totalement compris » (Nizet, et al., 2016, p. 45).

7 La question de l'engagement des étudiants est effectivement l'une des préoccupations majeures qui a émergé à l'issue de l'expérimentation PédagInnov. Selon le porteur du Projet PedagInnov, Luc Chevalier (Chevalier et Adjedj, 2014), et selon Jacquet-Faucillon (2014), un enseignant expérimentateur, l'un des principaux constats qui ressort de leur dispositif, a trait aux écarts qui se creusent entre les étudiants qui s'engagent et « jouent le jeu », et ceux qui ne s'engagent pas ou se désengagent, qui « ne jouent pas le jeu ». Les étudiants qui s'engagent préparent le cours en amont et appliquent leurs connaissances acquises en classe, ce qui participe à un apprentissage de meilleure qualité. En revanche, les étudiants qui ne s'engagent pas ont tendance à peu ou pas préparer le cours en amont, accentuant de fait leurs difficultés lors des exercices d'application en classe.

8 Comme nous venons de le voir, la classe inversée constitue une innovation pédagogique impliquant un changement de paradigme pédagogique qui peut être déstabilisant pour l'étudiant. Cette déstabilisation peut entrainer des attitudes attentistes ou de rejet de la part des étudiants et ainsi mettre à mal le bon fonctionnement du dispositif. L'engagement des étudiants dans la classe inversée constitue donc une nécessité. Notre questionnement est le suivant : quelles sont les sources d'influence de l'engagement des étudiants dans une innovation pédagogique de type classe inversée? En d'autres termes, comment favoriser l'engagement des étudiants dans une innovation pédagogique de type classe inversée?

Notre recherche s'inscrit dans le champ des recherches qualitatives en pédagogie universitaire, il s'agit d'une évaluation descriptive d'une innovation pédagogique. Dans un premier temps nous présenterons les éléments théoriques liés au concept d'engagement. Nous aborderons ensuite la méthodologie. Dans un troisième temps nous présenterons les principaux résultats obtenus. Enfin, nous discuterons les résultats. 


\section{Cadre théorique et problématique}

\subsection{Engagement}

\subsubsection{Définition de l'engagement}

Newmann, Wehlage et Lambourn (1992) définissent l'engagement comme l'investissement psychologique et l'effort dirigés vers l'apprentissage, la compréhension, la maîtrise des connaissances, le développement des compétences ou des habiletés qui sont attendues ${ }^{3}$. L'engagement se distingue donc de la motivation, même si les deux sont étroitement liés. La motivation constitue un état psychologique, un élément moteur de l'action, il s'agit d'une condition nécessaire à l'engagement (Pirot et De Ketele, 2000; Viau, 2009). L'engagement va néanmoins au-delà de la motivation, il est relié aux actes et actions de l'individu. L'engagement est donc à la fois d'ordre psychologique, mais aussi comportemental, comme le soulignent Pirot et De Ketele (2000) c'est « la décision volontaire de s'engager activement et profondément, mais aussi [...] la participation active dans les activités d'apprentissage » (p. 370).

\subsubsection{Dimensions de l'engagement}

11 Les recherches qui portent sur l'engagement l'envisagent selon plusieurs dimensions. Prégent, Bernard et Kozantis (2009) envisagent l'engagement selon deux dimensions : l'engagement comportemental et l'engagement cognitif. L'engagement comportemental est associé à la conduite de l'étudiant. Les auteurs citent par exemple la ponctualité en classe, la présence en classe, l'implication dans les situations d'apprentissages, la persévérance, la participation en classe ou encore la remise des travaux. Ce type d'engagement renvoie plutôt à des aspects quantitatifs tels que nommés par Pirot et De Ketele (2000), facilement observables et mesurables. L'engagement cognitif est plus complexe à évaluer, il s'agit de l'investissement intellectuel de l'étudiant, comme la concentration, l'attention ou ses stratégies d'apprentissage. Ce type d'engagement renvoie aux aspects qualitatifs décrits par Pirot et De Ketele (2000). Fredericks, Blumenfeld et Paris (2004) ajoutent une dimension émotionnelle qui fait référence aux réactions affectives et émotionnelles de l'étudiant en classe, à l'égard de l'école et de son enseignant.

\subsubsection{Catégorisation des sources d'influence de l'engagement}

Nous allons présenter ici trois recherches qui ont catégorisé les sources d'influence de l'engagement. Zhang, Hu et McNamara (2015) proposent une catégorisation en deux dimensions, à savoir les facteurs externes et les facteurs internes (personnels). Les facteurs externes sont liés aux éléments contextuels (la famille, les camarades, les amis, l'environnement) et aux éléments institutionnels (les cours, l'enseignant, l'accès à l'information, le soutien à l'étudiant). Les facteurs internes font référence aux éléments personnels, tels que l'intérêt, la motivation, la personnalité, la maîtrise de soi ou les facultés d'adaptation. Concernant les facteurs externes, Fredricks et al. (2004) et Günüç et Kuzu (2014) distinguent l'influence de l'institution (conditions matérielles, l'utilité du cours, la praticité du cours, le lien avec le vécu), de la classe (l'enseignant, les pairs, les règles, la part d'autonomie de l'élève, les activités) et de l'enseignant (rétroaction, compétence, pratique pédagogique, relation pédagogique). 


\section{2. États des lieux des recherches sur les sources d'influence de l'engagement}

13 Les recherches qui portent sur les sources d'influence de l'engagement montrent que l'enseignant y joue un rôle central. Des chercheurs de l'Université de Sherbrooke ont mené une recherche sur les prédicteurs de l'engagement et de la persévérance dans deux programmes universitaires basés sur l'apprentissage par problèmes (APP) et par projets (Bédard, Lison, Dalle et Boutin, 2010; Bédard, Lison, Dalle Côté et Boutin, 2012; Lison et al., 2011). 192 étudiants de premier cycle d'ingénieur se sont portés volontaires pour participer à cette étude. Premièrement, ils ont complété un questionnaire permettant de mesurer les principaux prédicteurs de l'engagement et de la persévérance dans leurs programmes respectifs. Deuxièmement, des focus groups ont été conduits avec 15 d'entre eux. Les résultats montrent que les trois variables qui prédisent le mieux l'engagement des étudiants sont 1 ) le soutien aux étudiants via des rétroactions constructives, une bienveillance de l'enseignant et l'organisation d'activités pédagogiques adaptées; 2) la contextualisation des apprentissages, avec des activités reliées au contexte professionnel; 3) et la pensée réflexive, en lien avec les nouveaux outils cognitifs acquis par les étudiants grâce à l'APP et aux activités pédagogiques proposées. Cette recherche montre l'effet positif des activités pédagogiques contextualisées et de haut niveau cognitif sur l'engagement, ainsi que du soutien de l'enseignant dans l'apprentissage.

14 Les résultats de cette étude pourraient être mis en perceptive avec d'autres recherches qui montrent le lien entre la motivation et l'engagement : l'étudiant s'engage parce que l'enseignant met en œuvre un « environnement motivant ». Les recherches de Wigfield et Eccles (2002) et de Wigfield et Waguer (2005) montrent en effet que les apprenants motivés de manière intrinsèque sont plus compétents et engagés dans leurs apprentissages que ceux qui ne sont pas motivés intrinsèquement. Cette étude va dans le sens des recherches de Blumenfeld, Kempler et Krajcik (2006) et de Deci (Deci, Koestner, et Ryan, 1999; Deci et Moller, 2005; Deci et Ryan, 2008) qui révèlent que lorsque les besoins en compétences, relationnelles, et en matière d'autonomie des apprenants sont satisfaits par les activités proposées par l'enseignant, la motivation augmente et conséquemment l'engagement de l'apprenant s'en trouve renforcé.

15 Ces recherches mettent en exergue un lien entre la pédagogie de l'enseignant (soutien, activités, feedback, contextualisation) et l'engagement de l'étudiant, ainsi qu'entre la motivation de l'étudiant et son engagement. Outre ces résultats, peu de recherches portent sur les sources d'influence de l'engagement et encore moins sur le lien entre les stratégies d'évaluation des apprentissages et l'engagement des étudiants. Certains guides et manuels pédagogiques en font mention sans le vérifier empiriquement. Falchikov (2005) par exemple, propose un ouvrage dans lequel elle fournit un compterendu scientifique des nombreuses modalités d'évaluation, avec un accent particulier sur le lien entre le choix de l'évaluation et l'engagement de l'étudiant. Dans le même sens, Nicol et Macfarlane-Dick (2006) expliquent comment l'évaluation formative peut aider l'étudiant à prendre en main son propre apprentissage, c'est-à-dire à s'engager. Enfin, dans leur guide, Angelo et Cross (1993) proposent d'associer la participation des étudiants dans leur processus d'évaluation pour augmenter leur engagement et la qualité de leurs apprentissages. 


\subsection{Conceptualisation} l'engagement selon les trois dimensions suivantes: l'engagement comportemental (l'agir), cognitif (le savoir apprendre) et affectif (le vouloir apprendre).

- L'engagement comportemental : cette dimension de l'engagement porte sur des éléments objectifs. Il est question ici 1) du temps et de l'effort fourni par l'étudiant, tel que le nombre d'heures de travail, la régularité, la présence en classe, la ponctualité, le respect des délais, la persévérance ou la planification de son travail; 2) sa participation en classe et dans les activités pédagogiques; et 3) ses interactions avec ses pairs et l'enseignant.

-L'engagement cognitif : cette dimension porte sur des éléments plus qualitatifs et subjectifs. Il s'agit des stratégies d'apprentissage cognitives et métacognitives de l'étudiant.

- L'engagement affectif: elle renvoie à la motivation extrinsèque (obtention d'une récompense ou évitement d'une punition) et intrinsèque de l'étudiant (enthousiasme, sentiment de compétence, valeur accordée), ainsi qu'à la relation interpersonnelle et pédagogique entretenue avec ses pairs et l'enseignant.

19 À partir des recherches pré-citées sur les sources d'influence de l'engagement (Fredricks et al., 2004; Günüç et Kuzu, 2014; Zhang, et al., 2015), nous envisageons trois principales sources d'influence: 1) internes, 2) pédagogiques et didactiques et 3) contextuelles :

- les sources d'influence internes proviennent de l'étudiant. Il s'agit du vouloir apprendre, savoir apprendre et l'agir;

- les sources d'influence pédagogiques et didactiques renvoient à ce qui se passe en classe. Il est question de l'explication du contrat pédagogique et didactique, l'animation du cours, les supports et ressources, l'aide apportée par l'enseignant, la relation pédagogique et l'évaluation;

- les sources d'influence contextuelles portent sur les caractéristiques du cours, à savoir le type de cours (CM, TP, TD), la matière et le niveau universitaire (L1, L2, L3, M1, M2).

Dans cet article, nous nous intéresserons uniquement aux sources d'influence pédagogique et didactique, c'est-à-dire à celles qui peuvent être contrôlées par l'enseignant. L'engagement est à envisager à la fois en tant que processus et en tant qu'état (Bryson, Cooper, et Hardy, 2010; Kahu, 2011). Le processus renvoie aux éléments qui influent sur l'engagement de l'étudiant, nous les nommons « sources d'influences ». Le résultat est l'engagement de l'étudiant (état) au niveau des trois dimensions: comportementale, cognitive et affective. Par exemple, en tant que processus, l'engagement affectif constitue une source d'influence interne, qui peut influer sur 
l'engagement cognitif et comportemental de l'étudiant. En tant qu'état, l'étudiant est ou n'est pas engagé au niveau affectif (cf. Figure 1).

Figure 1. Sources d'influence et dimensions de l'engagement.

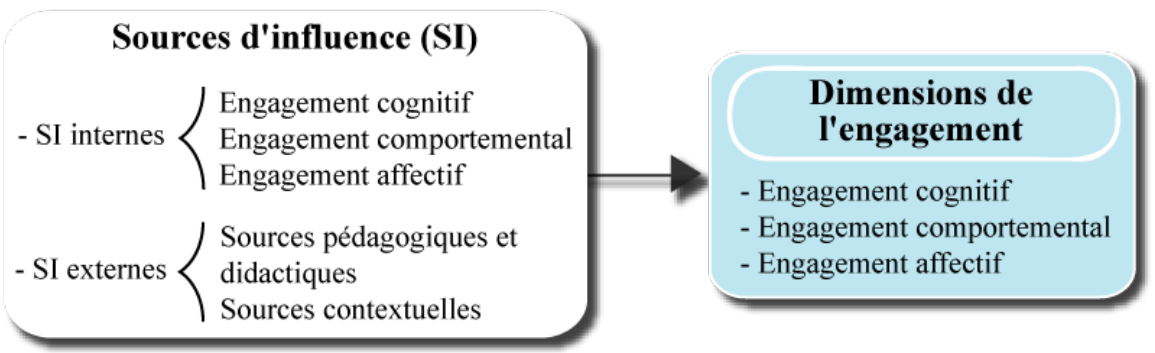

\section{Méthodologie}

\subsection{Instrumentation}

Pour répondre à notre question de départ sur les sources d'influence de l'engagement des étudiants dans une innovation pédagogique de type classe inversée, nous avons choisi de mener des entretiens semi-directifs auprès des étudiants et des enseignants.

Le guide d'entretien a été élaboré à partir des catégorisations identifiées dans la littérature (cf. Partie 2). À partir de la littérature, nous avions catégorisé trois principales sources d'influence de l'engagement: 1) internes; 2) pédagogiques et didactiques; 3 ) contextuelles. Comme mentionné précédemment, nous nous intéressons dans cet article uniquement aux sources d'influence pédagogiques et didactiques parce qu'elles peuvent être contrôlées par l'enseignant, contrairement aux sources internes (propres à l'étudiant) et contextuelles. La catégorie « sources d'influence pédagogiques et didactiques » se décompose elle-même en sous-catégories : a) l'explication du contrat pédagogique et didactique; b) l'aide apportée par l'enseignant; c) la relation pédagogique; d) les supports et ressources; e) l'animation du cours et f) l'évaluation. Le guide d'entretien a été élaboré sur la base de ces sous-catégories (cf. Tableau 1). Dans le tableau ci-dessous nous présentons également les questions développées en lien avec ces sous-catégories.

Tableau 1. Extrait du guide d'entretien.

\begin{tabular}{|c|c|c|}
\hline $\begin{array}{l}\text { Sources } \\
\text { pédagogiques et } \\
\text { didactiques }\end{array}$ & $\begin{array}{l}\text { Explicitation des règles/ } \\
\text { attentes }\end{array}$ & $\begin{array}{l}\text { - Comment l'enseignant vous a-t-il présenté le } \\
\text { fonctionnement de la pédagogie inversée? } \\
\rightarrow \text { Combien de temps ont été consacrés à } \\
\text { l'explication des règles de fonctionnement? } \\
\rightarrow \text { Est-ce que cette présentation présentait } \\
\text { clairement ce qui était attendu? Le travail que } \\
\text { tu devais fournir? Le rôle de l'enseignant? }\end{array}$ \\
\hline
\end{tabular}




\begin{tabular}{|l|l|}
\hline $\begin{array}{l}\text { Aide apportée/feedback/ } \\
\text { disponibilité de l'enseignant } \\
\text { dans la classe et en dehors }\end{array}$ & $\begin{array}{l}\text { - Comment l'enseignant t'apportait-il une } \\
\text { aide? } \\
\text { cours? En classe? } \\
\rightarrow \text { Quelle était sa disponibilité en amont du } \\
\text { Relation pédagogique }\end{array}$ \\
\hline rendu? Ces retours étaient-ils satisfaisants?
\end{tabular}

À travers le discours des acteurs, nous cherchions à :

1. identifier et analyser les sources d'influence pédagogiques et didactiques les plus récurrentes dans les discours des étudiants et des enseignants. Par exemple, selon les acteurs, la sous-catégorie " évaluation » joue-t-elle sur l'engagement des étudiants? Si oui, quel type d'évaluation semble avoir un effet positif sur leur engagement?;

2. identifier sur quelle(s) dimension(s) de l'engagement semble influer chacune des souscatégories. Nous rappelons ici qu’à partir de la littérature (cf. Partie 2), nous avions catégorisé trois dimensions de l'engagement: 1) l'engagement comportemental; 2) l'engagement cognitif; et 3) l'engagement affectif. Ainsi, par exemple, la sous-catégorie " évaluation » semble-t-elle davantage influer sur l'engagement comportemental, cognitif ou affectif de l'étudiant?

\subsection{Sujets}

Nous avons mené des entretiens avec 7 enseignants et 13 étudiants qui avaient expérimenté la classe inversée dans le cadre du Projet PedagInnov. Une seule femme faisait partie des 7 enseignants. Quatre enseignants nous ont fourni les adresses électroniques de leurs étudiants. Ces derniers ont ainsi été contactés personnellement 
par courrier électronique. 13 étudiants ont accepté l'entretien. Parmi ces 13 étudiants, il y a 7 femmes et 6 hommes. 9 étudiants sont inscrits dans une discipline des sciences dures (mécanique et mathématiques) et 4 étudiants sont inscrits dans une discipline des sciences humaines (espagnol et histoire).

Le rapport d'évaluation du Projet PedagInnov étant public, nous avons garanti l'anonymat des enquêtés en remplaçant leurs noms par une lettre de l'alphabet suivie d'un chiffre (ex : E1, E2). Il en a été de même pour le nom des enseignants (ex : A1, A2).

\subsection{Méthode d'analyse} an après l'expérimentation de la classe inversée. Il s'agissait d'entretiens semi-directifs d'une durée moyenne d'une heure. Les entretiens ont été retranscrits dans leur intégralité.

thématique a été réalisée à l'aide du logiciel de traitement de données qualitatives Nvivo. Une mesure de fidélité inter juge a été réalisée par la deuxième auteure de cet article sur l'ensemble du codage des entretiens. Un premier codage sous Nvivo a été réalisé par la première auteure de l'article. La deuxième auteure a ensuite apporté son regard critique sur le codage effectué. Les désaccords ont fait l'objet d'une discussion et d'un recodage au besoin.

Nous avons réalisé deux types d'analyse. Premièrement, notre analyse a consisté à identifier et analyser les sources d'influence pédagogiques et didactiques les plus récurrentes dans les discours. Ainsi, dans le discours des étudiants et des enseignants, nous avons comptabilisé les sources d'influence les plus fréquemment citées par les acteurs comme étant une source d'influence positive sur l'engagement des étudiants dans la classe inversée.

Deuxièmement, notre analyse a consisté à identifier et analyser le lien entre les sources d'influence et les dimensions de l'engagement. En d'autres termes, quelles dimensions de l'engagement semblaient les plus impactées en fonction de la source d'influence. Pour exemple, prenons l'extrait de l'entretien suivant: «je trouve que c'était plus compréhensible quand il commentait le diaporama, plutôt que juste les diapos " (E12). Ici, l'étudiant E12 fait un lien entre une source d'influence, à savoir le type de ressources (diaporama commenté) et une dimension de l'engagement, à savoir son engagement cognitif («plus compréhensible»). Ainsi, pour E12, le type de ressources utilisé par l'enseignant semble influer positivement sur son engagement cognitif. Dans le discours des enseignants, nous nous sommes appuyés sur leurs représentations et leurs constats personnels pour identifier les sources d'influence positives de l'engagement de l'étudiant dans la classe inversée. Par exemple, prenons cet extrait d'entretien suivant : "Le QCM [...] le côté ludique, ils aiment bien, ils me l'ont dit » (A2). Via les retours de ses étudiants ("ils me l'ont dit»), A2 a pu identifier un lien entre le type de ressources (" ludiques») et l'engagement affectif des étudiants («ils aiment bien»). Ainsi, pour A2, il semble y avoir un lien entre le type de ressources et l'engagement affectif des étudiants. 


\section{Résultats et analyse}

Le discours des étudiants et des enseignants montrent que trois principales sources d'influence pédagogique et didactique semblent influer positivement sur l'engagement de l'étudiant dans la classe inversée, à savoir 1) la posture d'accompagnateur de l'enseignant; 2) les ressources/supports utilisés et 3) l'évaluation.

\subsection{Posture d'accompagnateur}

À partir de l'analyse du discours des acteurs, nous avons synthétisé dans le tableau 2 les sources d'influence relatives à la posture d'accompagnateur de l'enseignant qui semblent influer positivement sur l'engagement des étudiants dans la classe inversée.

Tableau 2. Sources d'influence relatives à la posture d'accompagnateur qui semblent influer positivement sur l'engagement des étudiants

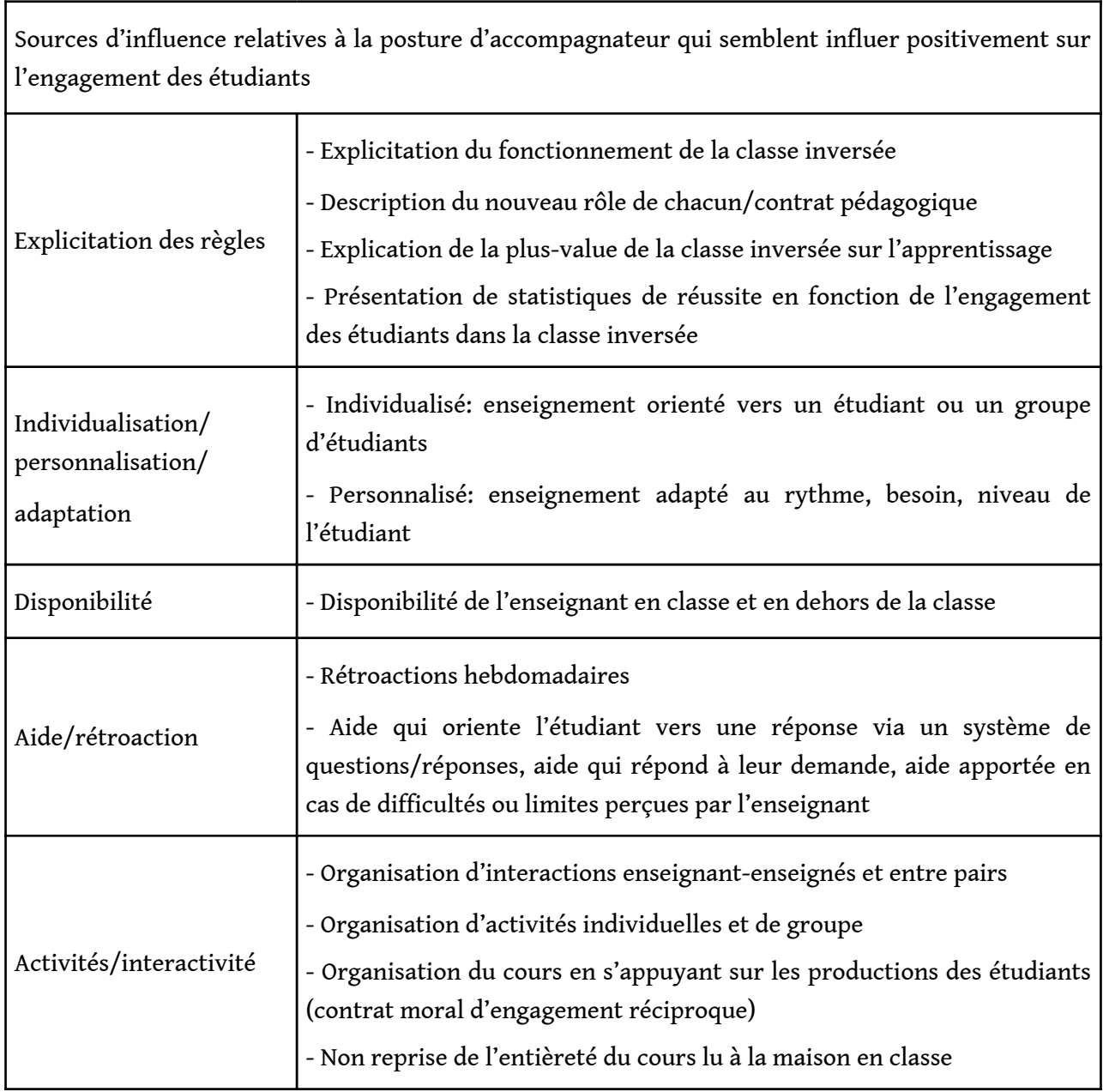




\begin{tabular}{|l|l|}
\hline & $\begin{array}{l}\text { - Évaluation de son dispositif de classe inversée pour vérifier son impact } \\
\text { et l'améliorer } \\
\text { Évaluation/ } \\
\begin{array}{l}\text { autoévaluation } \\
\text { dispositif }\end{array} \\
\text { - Récolte de preuves informelles : observation du comportement des } \\
\text { étudiants, qualité de leurs productions, notes, retours des étudiants sur } \\
\text { le dispositif. } \\
\text { - Récolte de preuves formalisées : passation d'un questionnaire }\end{array}$ \\
\hline
\end{tabular}

\subsubsection{Explicitation des règles}

Les 7 enseignants interrogés disent qu'ils explicitent le fonctionnement de la classe inversée à leurs étudiants, notamment le nouveau rôle de chacun et le nouveau contrat pédagogique : "je leur explique que je suis plus le coach, je vais les aider à apprendre, mais sur le fond c'est eux qui doivent faire le travail» (A2). Les enseignants considèrent l'explicitation du fonctionnement du dispositif comme l'une des clés pour favoriser l'engagement de leurs étudiants «je leur dis explicitement que c'est un dispositif de classe inversée. Je suis les conseils de la formation [pédagogique], c'est-à-dire que pendant la formation, on nous a bien dit d'être extrêmement clairs sur le dispositif, de bien le présenter, donc c'est ce que je fais» (A3). Quatre enseignants sur les 7 disent également expliquer aux étudiants pourquoi ils ont choisi d'organiser leur enseignement en classe inversée, en expliquant les limites de l'enseignement traditionnel et les bénéfices de la classe inversée sur l'apprentissage.

Onze étudiants sur 13 disent que leur enseignant a effectivement explicité le fonctionnement de la classe inversée lors du premier cours, incluant l'évolution du rôle de chacun «Il nous a surtout parlé de l'autonomie et de la régularité du travail qui devait être fourni » (E12), "Il nous a expliqué qu'il ne ferait pas de cours lui-même » (E11). L'explicitation des règles du jeu dès le départ constitue un cadrage qui est apprécié par les étudiants et qui semble influer positivement sur leur engagement affectif (motivation) «on est plus guidé, on sait vers où se diriger. On n'est pas lâché comme ça avec des documents à ne plus savoir quoi en faire» (E4), « tout était à notre disposition pour avoir une bonne note » (E5).

\subsubsection{Individualisation, personnalisation et adaptation}

Six enseignants disent qu'enseigner en classe inversée permet d'individualiser et de personnaliser l'apprentissage, permettant ainsi aux étudiants de bénéficier d'un enseignement plus en adéquation avec leurs besoins et leurs attentes. L'enseignement est plus individualisé dans le sens où le discours de l'enseignant peut être orienté uniquement vers un étudiant ou un groupe d'étudiants «Parfois, j'explique un cours pour un seul étudiant»(A3). Ces enseignants disent qu'ils s'approprient différemment l'espace classe : A4 et A6 se "balade[nt] dans l'amphi ", A3 s' "assoi[e] avec les petits groupes ». A4 va même jusqu'à déconstruire l'espace classe traditionnelle « ils sont très perturbés parce que je mets des classes en $U[. .$.$] je me mets systématiquement au fond de la classe » (A4).$

L'enseignement est aussi plus personnalisé, dans le sens où les enseignants connaissent mieux leurs étudiants. Ils disent qu'ils adaptent leurs discours et leurs ressources en fonction du niveau de connaissances et de compétences de l'étudiant: "Si c'est quelqu'un qui est bien bosseur, je vais pouvoir dire que c'est comme ce que l'on a vu au chapitre 
machin, et cela suffira comme indication "(A5). D'après le discours des enseignants, l'individualisation et la personnalisation de l'enseignement joueraient positivement sur l'engagement affectif des étudiants « Là, je m'assois avec les petits groupes, donc on a une proximité qui valorise l'étudiant [...] et donc il y a une relation de confiance à mon sens qui est plus importante. Je pense que cela doit jouer sur la motivation » (A3).

Huit étudiants mentionnent qu'ils apprécient cette individualisation des cours qui est induite par la classe inversée. Par exemple, lorsque l'enseignant s'appuie sur leurs productions pour construire le cours, les étudiants ont l'impression d'être partie prenante dans la création du cours, de le co-construire, ce qui semble avoir un effet sur leur engagement affectif (motivation) «Cela me semble plus motivant de se dire que l'on a un effet sur le cours" (E8) «le prof fait une liste des questions qu'il a eues, et pendant le CM, il en reparle bien. C'était sympa» (E2). Comme le souligne E8, il n'est plus question ici de cours génériques imposés par l'enseignant, mais d'un cours adapté en fonction des attentes et des besoins des étudiants «Ce n'est pas le même cours qui est fait chaque année. C'est vrai que c'est plus motivant" (E8). Les étudiants apprécient également la personnalisation du cours, qui se traduit par une évolution de la communication pédagogique "[lors des travaux de groupe] elle vient vraiment nous voir, cas par cas, elle ne reste pas à côté de son bureau [...] Elle vient nous voir pour voir comment on progresse, et où on en est » (E10). Quatre étudiants abordent spontanément cette nouvelle appropriation par l'enseignant de l'espace-classe. Les étudiants apprécient que l'enseignant se positionne en accompagnateur avec un discours personnalisé "il nous regardait vraiment, et il nous parlait [...] on voyait vraiment qu'il s'adressait à nous et pas juste à son tableau »(E9). La personnalisation semble avoir un effet positif sur leur engagement affectif.

\subsubsection{Disponibilité}

L'ensemble des enseignants interrogés mentionnent que la classe inversée implique une plus grande disponibilité dans et en dehors de la classe «certains m'envoient des trucs à l'avance avec des questions dedans, parce qu'ils ne savent pas répondre. J'essaye de répondre. Tout cela prend du temps » (A6). La disponibilité et l'implication de l'enseignant peut influer positivement sur l'engagement comportemental et cognitif des étudiants qui peuvent l'interpréter comme une sorte de contrat moral : «les élèves [...] sentent qu'il y a une implication, que l'on s'intéresse à eux, et du coup en retour, ils se sentent un peu obligés, pour certains, de participer » (A6).

Dans leurs discours, 6 étudiants ont désigné la disponibilité de l'enseignant comme une source d'influence positive. Il s'agit ici de la disponibilité de l'enseignant à répondre à leurs questions en classe et en dehors de la classe "si on avait des questions par mail, il y répondait toujours» (E1), «il prenait le temps de nous expliquer les choses en CM » (E5). Par exemple, dans le discours de $\mathrm{E} 7$, la disponibilité de l'enseignant à corriger un travail facultatif semble jouer sur l'effort fourni dans la réalisation de ce travail (engagement comportemental) : " $j$ 'en ai fait deux ou trois [des sujets d'examens], que je lui ai envoyés par mail. Il les a imprimés, il les a corrigés et il m'a envoyé les scans. Donc c'était pas mal. Cela donne l'impression de vraiment travailler» (E5). Par exemple, dans le témoignage de E9, nous pouvons observer que la disponibilité de l'enseignant à répondre à ses questions semble influer sur son implication dans l'apprentissage (engagement cognitif) : «il donnait plus envie de s'impliquer dans la matière, puisque le prof nous répond aussi si on a des questions. On n'est pas juste en train de travailler dans son coin, et quand on est en cours, on peut poser des 
questions » (E9). À travers le témoignage des 6 étudiants, nous observons un lien entre la disponibilité de l'enseignant et l'engagement affectif de l'étudiant «le fait de savoir que notre enseignant est toujours là pour nous répondre, que c'est lui qui nous envoie les cours, cela donne envie » (E12).

\subsubsection{Aide/rétroaction} étudiants. La classe inversée telle que mise en place par ces enseignants implique des rétroactions plus fréquentes que dans une situation de classe traditionnelle où les difficultés des étudiants se découvrent bien souvent le jour de la correction de l'examen «[avant je découvrais à l'examen que] les étudiants en savent moins que ce que je pensais qu'ils savaient [...] C'est une chose dont on ne se rend pas compte quand on fait un amphi, car il n'y a pas de feed-back» (A5). Dans le cadre de la classe inversée, les feedbacks sont plus nombreux. D'une part, la plupart des enseignants interrogés soumettent l'étudiant à un test de connaissance chaque semaine. D'autre part, les activités proposées en classe permettent également à l'enseignant de vérifier les acquis à chaque séance. À partir des entretiens, on remarque que l'aide apportée par les enseignants ne consiste pas à fournir la solution à l'étudiant. Les enseignants interrogés disent qu'ils poussent l'étudiant à trouver lui-même sa réponse. L'aide apportée prend alors différentes formes. Parfois, les enseignants apportent une aide qui répond à une demande exprimée par l'étudiant. L'aide est aussi spontanée, c'est-à-dire qu'elle se décide dans l'instant, en fonction des besoins ou de la demande et ne peut être anticipée par l'enseignant. Enfin, l'aide apportée est évolutive dans le temps, lorsque l'enseignant intervient davantage vers la fin du semestre qu'au début.

Six étudiants considèrent les rétroactions de l'enseignant comme une source d'influence positive sur leur engagement. Il s'agit ici des retours de l'enseignant sur leurs exercices, obligatoires ou facultatifs, envoyés en amont du cours. À travers le discours de ces 6 étudiants, nous observons que les rétroactions et l'aide apportés par l'enseignant semblent influer positivement sur les trois dimensions de l'engagement: affectif, cognitif et comportemental. Se sentant soutenus et épaulés dans leurs apprentissages, les étudiants s'engagent plus facilement dans la tâche " on sent aussi que Madame X est vraiment investie [...] elle nous motive [...] elle essaye de tout faire pour vraiment nous motiver, et nous tirer vers le haut" (E5). Par exemple pour E12, les corrections apportées par son enseignant semblent avoir un effet sur sa motivation (engagement affectif) et son engagement dans la tâche : "quand on lui envoyait [les exercices], il nous disait ce qu'il fallait revoir. C'était super sympa » (E12).

\subsubsection{Activités et interactivité}

41 En classe, l'ensemble des enseignants interrogés disent qu'ils mettent en place des activités de pédagogie active. Comme ils le spécifient dans leurs discours, ces activités influent sur l'engagement comportemental des étudiants parce qu'ils n'ont pas d'autres choix que d'être actifs en classe, de s'y investir « ils travaillent en équipe, et ils doivent aller chercher toutes les infos nécessaires» (A7), "Pour moi, c'est un des gros avantages du cours inversé, c'est qu'il met l'étudiant au pied du mur [...] il faut bosser » (A2).

Les entretiens de 9 étudiants montrent que l'interactivité et les activités d'apprentissage proposées en classe semblent surtout jouer sur leur engagement 
comportemental et cognitif. L'interactivité renvoie aux interactions enseignantenseignés "Elle interagit avec nous, et en fait, elle nous force à nous investir dans le cours" (E5), mais aussi aux interactions entre pairs «Je finissais par comprendre souvent en fin de séance, après avoir fait les exercices, et après avoir discuté avec mes camarades dans le groupe de quatre" (E13). Les activités d'apprentissage renvoient au travail personnel ou de groupe "on révisait le cours chez nous, et on l'appliquait en cours à chaque fois sur divers exercices. Je pense que c'est cela qui a rendu le sujet plus attrayant, et plus facile tout simplement à apprendre " (E5). Via l'organisation d'activités de pédagogie active et la multiplicité des interactivités horizontales (entre étudiants) et verticales (entre l'étudiant et l'enseignant), l'enseignant crée un espace qui favorise l'apprentissage par la confrontation et l'expérience pratique.

\subsection{6. Évaluation et autoévaluation du dispositif}

L'ensemble des enseignants interrogés sont entrés dans une dynamique d'autoévaluation de leur nouvelle pratique pédagogique et de leur dispositif de classe inversée. Cette autoévaluation a pour objectif d'obtenir des informations dans une visée d'amélioration. Pour obtenir des informations sur leur dispositif de classe inversée, ils s'appuient sur deux types de preuves : la récolte de preuves formelles et informelles. Quatre enseignants évaluent leur dispositif en observant, à travers le comportement des étudiants, leur degré d'adhésion. Par exemple, A1 analyse les statistiques de connexion des étudiants à la plateforme Moodle. A2 a observé le nombre décroissant d'étudiants qui venaient assister à son cours, lui donnant une indication sur la difficulté du contenu proposé. Cinq enseignants évaluent leur nouveau dispositif à travers la qualité des productions orales et écrites des étudiants «Je vois, en corrigeant, ce qui a mieux été et ce qui a été moins bien " (A1). Les notes obtenues par les étudiants constituent un autre indicateur de performance de la classe inversée pour 4 enseignants : "il y a une corrélation forte entre ceux qui se plantent à la fin, donc à l'examen, et ceux qui ne travaillent pas le cours en continu » (A5). Cinq enseignants ont demandé un retour de la part de leurs étudiants concernant le nouveau dispositif. Pour recueillir ces retours, 3 enseignants ont mis en place un questionnaire d'évaluation «Je fais une petite fiche en ligne avec un questionnaire [...] ça s'appelle Votre avis m'intéresse » (A4). A3 et A5, ont quant à eux interrogé quelques étudiants de manière informelle. L'évaluation de leur dispositif permet aux enseignants d'obtenir des informations sur le degré d'engagement des étudiants dans la classe inversée dans le but de trouver des solutions pour renforcer cet engagement.

\subsection{Ressources et supports}

À partir de l'analyse du discours des acteurs, nous avons synthétisé les sources d'influence relatives aux ressources/supports qui semblent avoir un effet positif sur l'engagement des étudiants dans un dispositif de classe inversée (cf. Tableau 3).

Tableau 3. Sources d'influence relatives aux ressources/supports

Sources d'influence relatives aux ressources/supports qui semblent influer positivement sur l'engagement des étudiants 


\begin{tabular}{|c|c|}
\hline $\begin{array}{l}\text { Qualité des } \\
\text { ressources et } \\
\text { supports }\end{array}$ & $\begin{array}{l}\text { - Type de ressources et supports : ludiques, les supports avec voix (diaporama } \\
\text { commenté, vidéo), diversifiés. } \\
\text { - Contenu : des supports contextualisés, mentionnant explicitement les } \\
\text { éléments importants, outils ou supports qui permettent à l'étudiant de } \\
\text { percevoir le travail ou l'opinion des autres étudiants }\end{array}$ \\
\hline $\begin{array}{ll}\text { Quantité } & \text { de } \\
\text { ressources } & \text { et } \\
\text { supports } & \end{array}$ & - Ni trop de documentation, ni trop peu \\
\hline
\end{tabular}

\subsubsection{Qualité}

L'ensemble des enseignants interrogés font mention dans leurs discours d'un lien entre les différents supports et ressources utilisés et l'engagement des étudiants. Premièrement, on observe un lien entre le type de support et l'engagement affectif de l'étudiant, influant par ricochet sur leur engagement comportemental "Ils n'étaient pas très nombreux à avoir regardé les vidéos, parce que les vidéos ne sont pas palpitantes, de leur point de vue ». Deuxièmement, dans leurs discours, ils font un lien entre le contenu des supports et l'engagement affectif et comportemental des étudiants, surtout lorsqu'ils sont contextualisés, c'est-à-dire lorsqu'ils renvoient à du connu, comme l'actualité ou des éléments de leur vie quotidienne "savoir comment fonctionnent les ménages au Moyen Âge [...] cela $a$ du sens à partir du moment où on se demande quelle est la répartition des rôles entre l'homme et la femme dans les ménages aujourd'hui [...] faire le lien avec ce qu'ils connaissent est absolument essentiel » (A4).

D'après le discours de 5 étudiants, le type d'outils et de supports de cours utilisés en classe semblent influer sur leur engagement. Le caractère ludique et la diversité des outils et des supports influent plutôt sur leur engagement affectif et par ricochet sur leur engagement comportemental «la façon dont il construisait son cours était assez ludique, à utiliser les commandes pour répondre aux QCM » (E2). Ils s'engagent dans la tâche (comportemental) parce que c'est ludique (affectif). Les outils ou supports qui permettent à l'étudiant de percevoir le travail, l'avis ou l'opinion des autres étudiants, influent plutôt sur leur engagement cognitif «il avait des captures d'écran des feuilles de travail, et c'est vrai que je pense que c'est utile de voir les erreurs que les autres ont pu faire, pour ne pas les refaire ensuite»(E1). Voir les opinions ou les erreurs des autres les amènent à réfléchir sur le sujet. Les étudiants font également mention de l'importance de la voix. E1 et E12 considèrent que le cours est plus compréhensible lorsque l'enseignant fournit le cours sous forme de diaporama commenté avec sa voix enregistrée «je trouve que c'était plus compréhensible quand il parlait, plutôt que juste les diapos» (E12). Comme le mentionnent les étudiants, la différence entre les éléments importants du cours est plus facilement identifiable à travers l'intonation de la voix. À partir du discours de trois étudiants, nous pouvons remarquer que la présentation visuelle des supports semble influer aussi sur l'engagement cognitif de l'étudiant « Visuellement, sur les Powerpoint, on voyait ce qui était important, et du coup, cela me permettait de retenir vraiment plus vite " (E9). Enfin, on remarque que l'engagement cognitif et affectif semble favorisé lorsque le cours est contextualisé « Le sujet était intéressant parce qu'on essayait de comprendre des choses que l'on utilise tous les jours » (E11). 


\title{
4.2.2. Quantité
}

\begin{abstract}
ressources nécessaires, fiables et directement en lien avec les connaissances à acquérir. Par exemple, pour E1, avoir accès à une documentation jugée exhaustive pour la compréhension du cours favorise son engagement cognitif dans la tâche "On allait sur l'ENT, on se connectait et on avait tous les documents pour comprendre, donc on n'avait pas besoin d'aller chercher sur internet"(E1). Toute la documentation étant mise à sa disposition, l'étudiant s'engage parce qu'il sait que sa réussite dépend uniquement de son appropriation de ces ressources. Pour E2, les liens hypertexte proposés par son enseignant semblent avoir joué sur son engagement comportemental «il mettait des vidéos sur YouTube, et il a aussi mis des pages Wikipédia [...] Quand il le mettait, j'allais voir, mais sinon, de moi-même, je ne le fais pas » (E2).
\end{abstract} comportemental. L'étudiant peut se sentir rassuré de disposer de l'ensemble des

\section{3. Évaluation}

À partir de l'analyse du discours des acteurs, nous avons synthétisé les sources d'influence relatives à l'évaluation qui semblent influer positivement sur l'engagement des étudiants dans la classe inversée (cf. Tableau 4).

Tableau 4. Les sources d'influence relatives à l'évaluation.

\begin{tabular}{|l|l|}
\hline $\begin{array}{l}\text { Sources d'influence relatives à l'évaluation qui semblent influer positivement sur l'engagement des } \\
\text { étudiants }\end{array}$ \\
\hline $\begin{array}{l}\text { Évaluation } \\
\text { multiple }\end{array}$ & $\begin{array}{l}\text { - QCM hebdomadaires notés pour la vérification des acquis et l'incitation au } \\
\text { travail en amont } \\
\text { - Demande de productions de haut niveau cognitif }\end{array}$ \\
\hline $\begin{array}{l}\text { Évaluation } \\
\text { formative }\end{array}$ & $\begin{array}{l}\text { - Rétroactions fréquentes et constructives } \\
\text { - Erreur perçue comme inhérente au processus d'apprentissage }\end{array}$ \\
\hline - Activités d'autoévaluation proposées \\
Eositive & $\begin{array}{l}\text { - Évaluation de la progression } \\
\text { - Récompense de l'investissement (points bonus) } \\
\text { - L'erreur n'est pas sanctionnée } \\
\text { - Suppression de la notation (validation obligatoire d'un pourcentage de } \\
\text { connaissances/compétences) } \\
\text { - Plusieurs tentatives possibles }\end{array}$ \\
\hline
\end{tabular}




\begin{tabular}{|l|l|}
\hline $\begin{array}{l}\text { Évaluation } \\
\text { transparente }\end{array}$ & $\begin{array}{l}\text { - Présentation des critères d'évaluation (ex: grille d'évaluation fournie en } \\
\text { amont) } \\
\text { vus en classe sont à l'examen, mention des chapitres qui feront l'objet de } \\
\text { l'évaluation) }\end{array}$ \\
\hline
\end{tabular}

\subsection{1. Évaluation multiple}

50 À partir du discours des enseignants, on remarque que l'évaluation est multiple. L'examen unique de fin de semestre n'est plus la norme. L'ensemble des enseignants interrogés proposent aux étudiants plusieurs évaluations continues au cours du semestre. Selon les enseignants, ces évaluations multiples obligent les étudiants à travailler régulièrement (engagement comportemental), ce qui a un effet positif sur la qualité de leur apprentissage (engagement cognitif) «ils sont en classe et ils sont obligés d'être actifs. Et après, il y a cette note à chaque activité, donc ils travaillent » (A3), «Les copies sont meilleures et les notes sont meilleures. Fatalement, puisqu'il y a davantage de contrôles continus » (A3).

51 À partir du témoignage de 9 étudiants, nous pouvons remarquer que l'évaluation fréquente influe positivement sur leur engagement cognitif et comportemental, même s'il ne s'agit que de quelques points bonus " Vu que la plupart des devoirs à rendre, les exercices à rendre étaient notés, c'est sûr que cela motive vraiment plus. Si ce n'est pas noté, je les fais, mais peut-être que je n'aurais pas autant de concentration» (E5). Les étudiants tendent à s'approprier cette contrainte pour se forcer à travailler régulièrement le cours. Les exercices sollicités en amont du cours peuvent même devenir de véritables défis personnels "je me disais toujours qu'il fallait que j'arrive à tout terminer » (E11), " Après, c'est personnel, j'avais vraiment besoin de réussir toute seule à chaque fois [...] cela développe quand même pas mal au niveau du travail acharné [...] et franchement, c'est sympa " (E2). On remarque à travers les entretiens des étudiants que les exercices hebdomadaires constituent pour eux des repères. Ils s'approprient les dates des rendus pour planifier leur travail sur la semaine et s'auto-évaluent à l'aide des exercices pour vérifier leurs apprentissages. Ils utilisent donc cette contrainte extérieure, que sont les exercices, pour créer leurs propres contraintes qui favorisent leur engagement.

\subsection{2. Évaluation formative}

L'évaluation formative s'apparente à l'aide et aux rétroactions régulières fournies par les enseignants (cf. section 4.1.4). L'ensemble des enseignants disent qu'ils font un retour sur les productions notées des étudiants et les accompagnent de manière continue dans leurs travaux en classe. Comme nous l'avons vu précédemment, le retour constructif et fréquent de l'enseignant sur les productions des étudiants, favorise leur engagement dans la tâche. L'erreur est perçue ici comme inhérente au processus d'apprentissage. Par exemple, concernant plus spécifiquement la production terminale demandée, A1 et A7 apportent leur aide et leurs conseils de manière continue aux groupes projet « quand je tourne au sein des différents groupes, je vois vraiment quand il y a un point bloquant [...] en fonction de cela j'adapte, et je fais de temps en temps un petit topo au tableau » (A1). 


\subsection{3. Évaluation transparente}

53 À travers le discours des enseignants, on constate que l'évaluation est explicite et transparente. Les critères sont définis et explicités aux étudiants dès les premières séances de cours. Par exemple, A2 et A6 pratiquent le contrat de confiance: ils expliquent aux étudiants qu'une partie des exercices vus en TD, corrigés ou non, seront proposés à l'examen. Une bonne partie de l'examen est donc connue de l'étudiant. A2 va plus loin en mentionnant à ses étudiants les chapitres du cours qui seront concernés par l'évaluation "pour chaque examen, je leur indiquais la liste des items concernés par l'examen, [...] Cela me paraissait une façon de les inciter à aller voir ce qu'ils étaient censés avoir fait pour l'examen» (A2). Un autre exemple, A1 et A7 fournissent leur grille d'évaluation $\mathrm{du}$ projet aux étudiants lors de la première séance, ils sont donc transparents sur les critères d'évaluation " je leur laisse le choix du sujet, en essayant d'être le plus clair possible sur les livrables [...] ils savent exactement ce qu'ils doivent faire, et ils construisent leur stratégie pour y arriver " (A7). Les étudiants savent exactement ce qui est attendu et peuvent orienter leur travail en conséquence.

54 L'analyse du discours des étudiants montre que leur contribution à la sélection du mode d'évaluation peut influer positivement sur leur engagement, comme c'est le cas avec $\mathrm{E} 4$ : « on a sélectionné ensemble les points les plus importants de ce qu'il y avait vraiment à connaître [...] les questions essentielles qui étaient susceptibles d'être posées à l'examen final [...] c'était vraiment les points importants qu'il fallait bien retenir [...] Personnellement, cela m'a permis de mieux apprendre mon cours " (E4). D'une part, l'étudiant sait sur quoi il sera évalué, ce qui influe sur son engagement comportemental et cognitif. D'autre part, la participation au choix de l'évaluation peut influer sur son engagement affectif (motivation).

\subsection{4. Évaluation positive}

Les enseignants interrogés abordent dans leur discours le caractère positif de l'évaluation. L'évaluation positive a pour objectif d'influer positivement sur l'engagement affectif (motivation) des étudiants. Par exemple A2 donne la possibilité aux étudiants de passer plusieurs fois le test hebdomadaire " $\mathrm{Ce}$ sont des exercices en ligne, c'est le système WIMS, et avec le mode de notation que l'on a installée, ils ont le droit à autant d'essais qu'ils veulent, et s'ils réussissent, c'est réussi. S'ils ratent, ils ne sont pas pénalisés de rater " (A2). A6, quant à lui récompense l'implication de l'étudiant par l'attribution de points bonus « les points bonus existent. J'essaye de les pratiquer un peu partout. C'est pour les motiver à faire le travail préparatoire » (A6). L'erreur n'est pas sanctionnée «Le principe est qu'ils font un travail préparatoire. Peu importe ce qu'ils ont mis dedans [...] Et donc je compte le nombre de travaux préparatoires, et il y a un bonus de 0,1 à 0,2 point par travaux rendus" (A6). A6 valorise la progression de l'étudiant au cours du semestre. A7 a même supprimé la notation de son $\mathrm{QCM}$, dans le but de redonner du sens à l'apprentissage « je fais un QCM qu'ils doivent valider absolument [...] ils doivent avoir 95 sur 100 [...] Tant qu'ils n'atteignent pas le score voulu, ils le refont [...] s'ils l'ont, ils ont le droit de continuer » (A7).

D'après le discours des étudiants, on remarque que le type d'évaluation peut effectivement jouer sur leur engagement. Par exemple, E1 s'engage au niveau cognitif et comportemental parce qu'une partie de l'évaluation porte sur des éléments connus "[Avoir $60 \%$ d'exercices issus des TD] [...] cela nous obligeait aussi à refaire beaucoup les TD, à comprendre la méthode» (E1). Il en est de même pour E12 qui s'engage parce que le 
système d'évaluation proposé permet d'obtenir facilement des points bonus "Je trouve que sa méthode est géniale, parce qu'on est obligé de refaire plusieurs fois les QCM [...] si on a 20 sur tous les QCM, on a ces deux points [...] A chaque fois, il faut les faire, les refaire et les refaire pour avoir 20, et quand on a faux, il nous donne la réponse. Et la réponse, c'est du cours. Du coup, plus on le refait, plus on apprend son cours" (E12). Dans les deux cas, l'évaluation est ressentie par les étudiants comme positive plutôt que comme sanctionnante.

\section{Analyse}

\subsection{Leviers de l'engagement}

Pour résumer ici, les résultats de notre étude montrent trois principales sources d'influence pédagogique et didactique qui semblent jouer sur l'engagement des étudiants dans la classe inversée : 1) la posture d'accompagnateur de l'enseignant; 2) les ressources/supports utilisés; et 3) l'évaluation. Premièrement, la posture d'accompagnateur (cf. Tableau 2) implique l'explicitation des règles de fonctionnement de la classe inversée, une individualisation et une personnalisation de la formation, une grande disponibilité dans et en dehors de la classe, l'apport de feedbacks réguliers et constructifs, l'organisation d'activités de pédagogie active de haut niveau cognitif et la mise en place d'un système d'évaluation de son dispositif pédagogique dans une visée d'amélioration. Deuxièmement, au niveau des supports et ressources (cf. Tableau 3), l'engagement des étudiants semble favorisé lorsque les supports/ressources sont en quantité suffisante et de qualité : ludiques, diversifiés, contextualisés, supports avec voix, éléments importants mis en exergue, productions des autres étudiants montrés en exemple. Troisièmement, l'engagement est favorisé lorsque l'évaluation est multiple, formative, transparente et positive (cf. Tableau 4). La figure 2, résume ces trois principales sources d'influence de l'engagement de l'étudiant dans la classe inversée que nous avons pu identifier à travers le discours des acteurs.

Figure 2. Les principales sources d'influence pédagogiques et didactiques de l'engagement des étudiants dans une classe inversée

\section{Sources d'influence pédagogiques et didactiques}

\section{Posture d'accompagnateur}

- Explicitation des règles

- Individualisation, personnalisation et adaptation - Disponibilité
- Aide/feedback

- Activités et interactivité

- Evaluation et autoévaluation du dispositif

Ressources et supports

- Qualité

- Quantité adéquate
- Multiple

- Formative

- Transparente

- Positive 
58 Les résultats de notre recherche montrent que les étudiants s'engagent pour trois principales raisons : 1) ils se sentent soutenus; 2) contraints; 3) et acteurs de leur réussite. Premièrement, les étudiants s'engagent parce qu'ils se sentent soutenus et épaulés dans leurs apprentissages. C'est le cas lorsque l'enseignant individualise, personnalise et adapte son cours, se rend disponible, apporte une aide et des rétroactions, propose des ressources et des supports de qualité, met en place une évaluation formative et positive, et fait participer les étudiants à l'évaluation de son dispositif de classe inversée. Les étudiants sont d'autant plus motivés lorsqu'ils perçoivent que leur enseignant s'implique dans leur réussite. Ce soutien ressenti tend à favoriser l'engagement affectif des étudiants (motivation), influant de fait sur leur engagement cognitif et comportemental.

Deuxièmement, les étudiants s'engagent parce qu'ils n'ont pas d'autres choix. C'est le cas lorsque l'enseignant met en place des activités de pédagogie active et sollicite des travaux multiples (évaluation multiple). Ces activités qui font partie intégrante de la classe inversée favorisent leur engagement comportemental. Ils n'ont pas d'autres choix que de s'investir et de s'impliquer dans les activités proposées par l'enseignant s'ils souhaitent valider leur module.

60 Troisièmement, les étudiants s'engagent parce qu'ils se sentent acteurs de leur réussite, le contrat pédagogique est transparent. Le contrat est transparent lorsque l'enseignant explicite clairement le fonctionnement de la classe inversée avec l'évolution du rôle de chacun, lorsqu'il fournit des ressources exhaustives et lorsqu'il met en place une évaluation transparente. La transparence du contrat pédagogique tend à favoriser leur engagement cognitif. Tout est mis à leur disposition pour qu'ils développent des apprentissages significatifs. Dès lors, la réussite n'est plus tributaire des implicites et de la chance. Ainsi, les étudiants s'engagent parce qu'ils perçoivent que la réussite dépend principalement d'eux, c'est-à-dire de leur volonté et de leur capacité à jouer le jeu attendu.

61 Nos résultats vont dans le sens des études de Lison et al. (2011), de Bédard et al. (2010) et de Bédard et al. (2012), plus spécifiquement en ce qui concerne le lien entre le niveau d'engagement des étudiants et les éléments-support (aide) apportés par l'enseignant. $\mathrm{Au}$ sein du projet PedagInnov, les enseignants apportent une attention particulière à l'aide apportée aux étudiants, notamment via leur posture d'accompagnateur, l'usage de ressources adaptées et la mise en place d'une évaluation bienveillante. Les résultats de la recherche de Lison et al. (2011) mentionnent également la contextualisation des apprentissages et la pensée réflexive comme un facteur d'engagement. Notre recherche va également dans ce sens, les supports contextualisés et les activités de haut niveau cognitif semblent influer positivement sur l'engagement des étudiants. Concernant le lien entre la motivation et l'engagement abordé par certains auteurs (Deci et Ryan, 2008; Wigfield et Eccles, 2002; Wigfield et Waguer, 2005), notre recherche montre aussi qu'un étudiant motivé s'engage plus facilement dans le dispositif. Enfin, par rapport au lien entre l'évaluation et l'engagement mentionné dans certains guides pédagogiques (Angelo et Cross, 1993; Falchikov, 2005; Nicol et Macfarlane-Dick, 2006), les résultats de notre recherche semblent effectivement confirmer ce lien. 


\subsection{Garder l'équilibre pour favoriser l'engagement}

62 À partir des résultats de notre recherche, on observe que la mise en place de la classe inversée nécessite un équilibre constant pour favoriser des apprentissages de haut niveau tout en maintenant l'engagement des étudiants. Six équilibres ont été identifiés (cf. Figure 3).

Figure 3. Garder l'équilibre pour favoriser l'engagement de l'étudiant dans la classe inversée

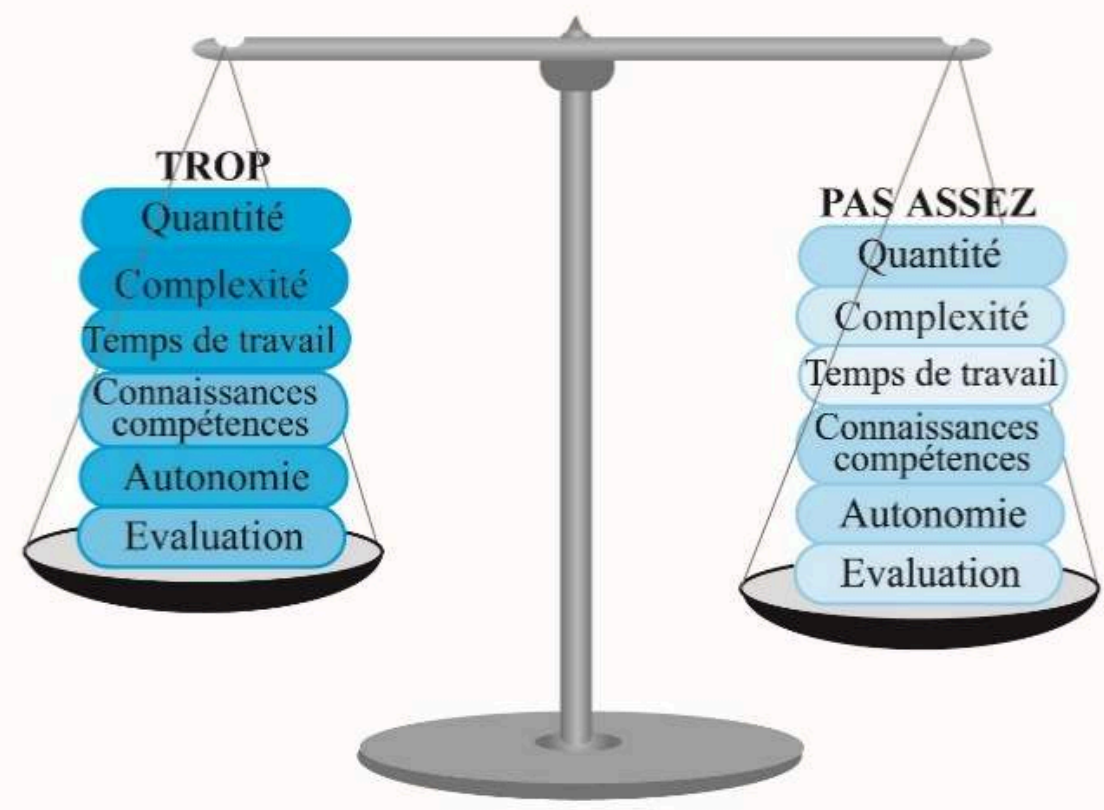

Un premier équilibre est à maintenir au niveau des quantités. Trop peu de ressources fournies à l'étudiant ne lui permettent pas de disposer de suffisamment de contenu pour se l'approprier. À l'opposé, trop de ressources risquent de le submerger et donc de le démotiver (désengagement affectif). Ce désengagement affectif aurait alors une incidence directe sur les deux autres dimensions de l'engagement.

Un deuxième équilibre porte sur la complexité des tâches demandées. Des exercices trop simples ne permettent pas d'atteindre des niveaux d'apprentissage en profondeur. À l'opposé, des exercices trop complexes créent chez l'étudiant un sentiment d'incompétence qui favorise son désengagement cognitif.

Le troisième équilibre a trait au temps de travail en autonomie. Si le temps de travail requis est trop court, il ne permettra pas à l'étudiant de développer des apprentissages en profondeur. À l'inverse, si le temps de travail est trop long, l'étudiant risque de se démotiver ou de ne pas réussir à organiser son planning en conséquence, et donc de se désengager.

Un quatrième équilibre est à prévoir entre les activités qui favorisent le développement de connaissances disciplinaires et les activités qui favorisent le développement de compétences transversales. Si c'est uniquement l'accroissement des connaissances qui est visé, la classe inversée ne permettra pas de développer des apprentissages 
significatifs, c'est-à-dire de haut niveau cognitif. À l'opposé, si les activités de pédagogie active sollicitent trop les compétences transversales des étudiants (ex : prise de parole en public lors d'un débat), ceux qui n'ont pas eu l'occasion de les développer dans d'autres circonstances, risquent de se désengager par manque de compétences.

Le cinquième équilibre porte sur l'autonomie. Si la classe inversée est trop cadrée et prescriptive, l'étudiant ne disposera pas de l'espace nécessaire pour développer son autonomie. À l'inverse, si trop d'autonomie est donnée à l'étudiant, il risque de se désengager par manque de cadres et de percevoir l'autonomie comme une absence d'aide plutôt que comme une compétence à développer.

Enfin, le dernier équilibre a trait à l'évaluation. Si elle est uniquement sanctionnante, l'étudiant ne disposera pas de suffisamment de rétroactions pour progresser dans son apprentissage et tendra, par ailleurs, à s'engager principalement par la crainte de l'échec. Si l'évaluation est uniquement formative, non notée, l'étudiant risque de ne pas bénéficier d'assez de contraintes pour s'obliger à s'engager.

\section{Conclusion}

La classe inversée constitue une innovation pédagogique impliquant un changement de paradigme pédagogique qui peut être déstabilisant pour l'étudiant. Cette déstabilisation peut entrainer des attitudes attentistes ou de rejet de la part des étudiants et ainsi mettre à mal le bon fonctionnement du dispositif. L'engagement des étudiants dans la classe inversée est donc primordial. Notre questionnement était le suivant: quelles sont les sources d'influence de l'engagement des étudiants dans une innovation pédagogique de type classe inversée? En d'autres termes: comment favoriser l'engagement des étudiants?

L'état des lieux des recherches sur l'engagement, nous a permis de catégoriser trois principales sources d'influence de l'engagement: 1) internes, 2) pédagogiques et didactiques et 3) contextuelles. Dans cet article, nous avons fait le choix de nous intéresser uniquement aux sources d'influence pédagogique et didactique, une source d'influence controlable par l'enseignant. Ainsi, notre premier objectif visait à identifier et analyser les sources d'influence pédagogiques et didactiques les plus récurrentes dans les discours des étudiants et des enseignants.

71 L'état des lieux des recherches sur l'engagement, nous a également permis de catégoriser les trois dimensions de l'engagement: l'engagement comportemental (l'agir), cognitif (le savoir apprendre) et affectif (le vouloir apprendre). Ainsi, notre deuxième objectif visait à identifier et analyser le lien entre les sources d'influence et les dimensions de l'engagement. En d'autres termes, quelles dimensions de l'engagement semblaient les plus impactées en fonction de la source d'influence.

Pour répondre à ces deux objectifs, nous avons mené des entretiens semi-directifs auprès de 13 étudiants et de 7 enseignants qui ont testé la classe inversée.

73 Les résultats montrent trois principales sources d'influence pédagogique et didactique sur l'engagement des étudiants, à savoir la posture d'accompagnateur, les ressources/ supports et l'évaluation. La classe inversée est une pédagogie d'équilibriste dans le sens où l'enseignant doit constamment trouver un équilibre pour favoriser des apprentissages de haut niveau tout en maintenant l'engagement des étudiants. Un équilibre est à trouver concernant la quantité des ressources à fournir, le degré de 
complexité des tâches demandées, le temps de travail requis en amont, la balance entre le développement de connaissances disciplinaires et transversales, le cadrage et l'autonomie, l'évaluation formative et sanctionnante.

74 La taille restreinte du groupe d'étude ne permet cependant pas d'étendre l'analyse à l'ensemble des dispositifs de classe inversée. Néanmoins, ces résultats pointent l'importance d'évaluer les dispositifs innovants, de faire de la recherche en pédagogie universitaire, de manière à pouvoir identifier les leviers pour améliorer les dispositifs et les pratiques pédagogiques. Enfin, il apparaît essentiel dans le contexte actuel d'accorder de l'importance à la diffusion des résultats d'expérimentations et d'innovations mises en place dans l'enseignement supérieur.

\section{BIBLIOGRAPHY}

Angelo, T. A. et Cross, K. P. (1993). Classroom assessment techniques: A handbook for college teachers. San Francisco, CA : Jossey-Bass Publishers.

Béchard, J.-P. et Pelletier, P. (2001). Développement des innovations pédagogiques en milieu universitaire : un cas d'apprentissage organisationnel. Dans D. Raymond (dir.), Nouveaux espaces de développement professionnel et organisationnel. Sherbrooke, Canada : Éditions de CRP.

Bédard, D. (2006). Enseigner autrement, oui mais pourquoi et comment? Le cas d'un cours universitaire du premier cycle. Dans N. Rege Colet et M. Romainville (dir.), La pratique enseignante en mutation à l'université. Bruxelles, Belgique : De Boeck.

Bédard, D. et Béchard, J.-P. (dir.) (2009). Innover dans l'enseignement supérieur. Paris, France : Presses Universitaires de France.

Bédard, D., Lison, C., Dalle, D. et Boutin, N. (2010). Predictors of student's engagement and persistance in an innovation PBL curriculum : applications for engineering education. International Journal of engineering education, 26(3), 511-522.

Bédard, D., Lison, C., Dalle, D., Côté, D. J. et Boutin, N. (2012). Problem-based and project-based learning in engineering and medecine: determination of students' engagement and persistance. Interdisciplinary journal of problem-based learning, 6(2).

Berthiaume, D. (2011). Innovation et pédagogie universitaire. Dans M.-J. Barbot et L. Massou (dir.), TIC et métiers de l'enseignement supérieur. Émergences, transformations. Nancy, France : Presses universitaires de Nancy.

Blumenfeld, P. C., Kempler, T. M., et Krajcik, J. S. (2006). Motivation and cognitive engagement in learning environments. Dans R. K. Sawyer (dir.), The Cambridge handbook of learning sciences (p. 475-488). NewYork, NY : Cambridge University Press.

Bryson, C., Cooper, G. et Hardy, C. (2010, décembre). Reaching a common understanding of the meaning of student engagement. Communication présentée au Society of Research into Higher Education Conference, Celtic Manor, Wales, Royaume Uni.

Chevalier, L. et Adjedj, P. J. (2014). Une expérience de classe inversée à Paris-Est. Technologie, 194, 26-37. 
Coulon, A. (1997). Le métier d'étudiant. L'entrée dans la vie universitaire. Paris, France : Presses Universitaires de France.

Dalle, D. et Lachiver, G. (2003). L'intégration des formations par problèmes et par projets dans les programmes de génie électrique : un défi pour les étudiants et pour les professeurs. Dans A. Balleux (dir.), La formation à l'enseignement professionnel: identité, enjeux et perspectives. Actes du 20 e congrès de l'Association internationale de pédagogie universitaire (AIPU), Université de Sherbrooke, 27-30 mai 2003. Sherbrooke : Université de Sherbrooke.

Deci, E. L. Koestner, R. et Ryan, R. M. (1999). A meta analytic review of experiments examining the effects of extrinsic rewards on intrinsic motivation. Psychological Bulletin, 125(6), 627-668.

Deci, E. L. et Moller, A. C. (2005). The concept of competence : A starting place for understanding intrinsic motivation and self determined extrinsic motivation. Dans J. A. Elliot et C. S. Dweck (dir.), Handbook of competence and motivation. New York, NY : The Guilford Press.

Deci, E. L. et Ryan, R. M. (2008). Facilitating optimal motivation and psychological well being across life's domains. Canadian Psychology, 49(1), 14-23.

Dumont, A. et Berthiaume, D. (2016). La pédagogie inversée. Louvain-la-neuve, Belgique : De Boeck supérieur.

Falchikov, N. (2005). Improving assessment through student Involvement. Practical solutions for aiding learning in higher and further education. Londres, Royaume Uni : Taylor \& Francis e-Library.

Fredricks, J. A., Blumenfeld, P. et Paris, A. (2004). School engagement: Potential of the concept, state of the evidence. Review of Educational Research, 74, 59-109.

Gerard, L. (2018). L'engagement des étudiants dans la pédagogie inversée. Projet PedagInnov. Rapport synthétique. http://idea.univ-paris-est.fr/fr/a-la-une/document-3139.html

Günüç, S. et Kuzu, A. (2014). Factors influencing student engagement and the role of technology in student engagement in higher education : Campus- class- technology theory. Turkish online journal of qualitative inquiry, 5(4), 86-113.

Jacquet-Faucillon, B. (2014). Classe inversée... de l'efficacité de la méthode... [Présentation PowerPoint]. www.univ-paris-est.fr/fichiers/07\%20-\%20Efficacité-méthode_190914.pdf

Kahu, E. R. (2011). Framing student engagement in higher education. Studies in higher education, 38(5), 758-773.

Lebrun, M. (2016). La classe inversée au confluent de différentes tendances dans un contexte mouvant. Dans A. Dumont et D. Berthiaume (dir.), La pédagogie inversée. Louvain-la-neuve, Belgique : Deboeck supérieur.

Lison, C., Bédard, D., Boutin, N., Côté, D. J., Dalle, D. et Lefebvre, N. (2011). L'engagement et la persévérance des étudiants dans trois programmes innovants de premier cycle en génie et en médecine. Revue des sciences de l'éducation, 37(1), 83-104.

Newmann, F. M., Wehlage, G. G. et Lambourn, S. (1992). The significance and sources of student engagement. Dans F. M. Newmann (dir.), Student engagement and achievement in the American secondary schools (p. 11-39). New York, NY : Teachers College Press.

Nicol, D. J. et Macfarlane-Dick, D. (2006). Formative assessment and self-regulated learning : a model and seven principles of good feedback practice. Studies in Higher Education, 31(2), 199-218.

Nizet, I., Galiano, O. et Meyer, F. (2016). Vers un cadrage théorique pour comprendre la classe inversée. Dans A. Dumont et D. Berthiaume (dir.), La pédagogie inversée (p. 39-47). Louvain-laNeuve, Belgique : De Boeck. 
Pirot, L. et De Ketele, J.-M. (2000). L'engagement académique de l'étudiant comme facteur de réussite à l'université. Étude exploratoire menée dans deux facultés contrastées. Revue des sciences de l'éducation, XXVI(2), 367-394.

Prégent, R., Bernard, H. et Kozanitis, A. (2009). Enseigner à l'université dans une approcheprogramme. Québec, Canada : Presses internationales Polytechnique.

Viau, R. (2009). L'impact d'une innovation pédagogique : au-delà des connaissances et des compétences. Dans D. Bédard et J.-P. Béchard (dir.), Innover dans l'enseignement supérieur. Paris, France : Presses universitaires de France.

Wigfield, A. et Eccles, J. S. (2002). Development of achievement motivation. San Diego, CA : Academic Press.

Wigfield, A. et Waguer, A. L. (2005). Competence, motivation and identity development during adolescence. Dans J. A. Elliot et S. C. Dweck (dir.), Handbook of competence and motivation. New York, NY : The Guilford Press.

Zhang, Z., Hu, W. et McNamara, O. (2015). Undergraduate student engagement at a Chinese university: a case study. Educationnal Assessment, Evaluation and Accoutability, 27(2), 105-127.

\section{NOTES}

1. Le porteur du Projet était M. Luc Chevalier, directeur de l'École Supérieure d'Ingénieurs (ESIPE) de l'Université Paris-Est Marne-la-Vallée (UPEM). Les 10 enseignants qui ont testé la classe inversée sont issus de l'UPEM, de l'Université Paris-Est Créteil (UPEC) et de l'École des Ponts Paris Tech.

2. Massive Open Online Course.

3. "The student's psychological investment in and effort directed towards learning, understanding, or mastering the knowledge, skills or crafts that academic work is intended to promote » (Newmann, Wehlage et Lambourn, 1992, p. 12).

\section{ABSTRACTS}

en

The project PedagInnov is an experimentation of the flipped classroom in higher education. It is part of a ministerial educational project Individualisation, diversification, evaluation and mentoring (IDEA), which has been developed since 2012 by the COMUE of the University ParisEst. Ten academics have accepted to change their traditional courses into flipped classrooms. We have focused on the following question: how could we encourage students' engagement in an educational innovation such as the flipped classroom? To answer the question, we interviewed 13 students and 7 academics who have chosen to test the flipped classroom. We have identified three main sources of implication of students: the posture of the mentor teacher, resources and supports, and evaluation.

fr 
Le Projet PedagInnov est une expérimentation de la classe inversée dans l'enseignement supérieur. Il s'inscrit dans le cadre de l'IDEFI Individualisation, Diversification, Évaluation et Accompagnement (IDEA) porté par la COMUE Université Paris-Est depuis 2012. Dix enseignants universitaires volontaires ont accepté d'organiser leurs cours selon le principe de la classe inversée. Notre question de recherche fut la suivante: comment favoriser l'engagement des étudiants dans une innovation pédagogique de type classe inversée? Pour ce faire, nous avons mené des entretiens semi-directifs auprès de 13 étudiants et de 7 enseignants qui avaient testé la classe inversée. Au niveau des sources d'influence pédagogiques et didactiques, nous avons pu identifier trois principales sources qui influent sur l'engagement des étudiants: la posture d'accompagnateur, les ressources et supports et l'évaluation.

\section{INDEX}

Mots-clés: enseignement supérieur, pédagogie inversée, classe inversée, engagement, innovation pédagogique, étudiants

\section{AUTHORS}

\section{LAETITIA GERARD}

Consultante internationale, gerard.laetitia@gmail.com

\section{ARIADNA AYALA RUBIO}

Conseillère pédagogique (IDEA-Université Paris-Est) 